\title{
Advances in terahertz communications accelerated by photonics
}

Tadao Nagatsuma ${ }^{1 *}$, Guillaume Ducournau ${ }^{2}$, and Cyril C. Renaud ${ }^{3}$

${ }^{1}$ Graduate School of Engineering Science, Osaka University, 1-3 Machikaneyama, Toyonaka, Osaka 560-8531, Japan

2Institut d'Electronique de Microélectronique et de Nanotechnologie (IEMN), UMR CNRS 8520, Université de Lille 1, 59652, Villeneuve d'Ascq CEDEX, France ${ }^{3}$ Department of Electronic and Electrical Engineering, University College London, London, WC1E 7JE, U.K.

*e-mail: nagatuma@ee.es.osaka-u.ac.jp

Almost 15 years have passed, since the initial demonstrations of terahertz (THz) wireless communications were made using both impulse and continuous waves. THz technologies have recently gained greater interest and expectations to meet an ever-increasing demand for the speed of wireless communications. This article reviews a latest trend of $\mathrm{THz}$ communications research focusing on how photonics technologies have played a key role in the development of first-age $\mathrm{THz}$ communication systems and how they compare with other competitive technologies such as THz transceivers enabled by electronic devices as well as free-space lightwave communications. 


\section{Trends in wireless communications}

It is known that data traffic is increasing exponentially with Internet Protocol (IP) traffic expected to reach over 130 Exabytes per month by $2018^{1}$. The fastest growing part of that increase is on wireless channels, as mobile users increasingly make use of online services. Such an increase in the network capacity requires much higher wireless transmission rates in numerous connection links between each base station, between a base station and an end-user device, between each end-user device, etc. The prospective data rate for wireless communications in the market place will be $100 \mathrm{Gbit} / \mathrm{s}$ within 10 years ${ }^{2}$. Historically, since the first microwave wireless link developed by G. Marconi in early $20^{\text {th }}$ century, carrier frequencies used for wireless communications have been increasing ${ }^{3,4}$ to meet bandwidth requirements, up to the recent development of wider spectral bands at millimetre-wave (MMW) frequencies, such as $60 \mathrm{GHz}$, and $70 \mathrm{GHz} \sim 95 \mathrm{GHz}{ }^{5}$. However, the total allocated bandwidth is less than $7 \mathrm{GHz} \sim 9 \mathrm{GHz}$ which will ultimately limit the total throughput of the channel to an insufficient level for the increasing demand.

It is obvious that the use of even higher carrier frequency in the $\mathrm{THz}$ range $(0.1$ $\mathrm{THz} 10 \mathrm{THz}$ ) is required when the bandwidth is at a minimum several tens of GHz. The initial demonstrations of $\mathrm{THz}$ wireless communications were conducted using both impulse and continuous waves, which were generated from photoconductors and photodiodes excited by pulse lasers and intensity-modulated lasers, respectively ${ }^{6-8}$. The latter continuous-wave wireless link, which employs a $120-\mathrm{GHz}$ band, is the first commercial $\mathrm{THz}$ communication system with an allocated bandwidth of $18 \mathrm{GHz}(116 \mathrm{GHz} 134 \mathrm{GHz})$, which offers $10 \mathrm{Gbit} / \mathrm{s}$ with an On-Off-Keying (OOK) modulation and $20 \mathrm{Gbit} / \mathrm{s}$ with a Quadrature Phase Shift Keying (QPSK) modulation at a transmission distance actually demonstrated of over $5 \mathrm{~km}^{9,10}$. Now, lots of worldwide research groups have developed 
communication links at frequencies over $100 \mathrm{GHz}$. In particular, above $275 \mathrm{GHz}$, there is a possibility to employ extremely large bandwidth of over $50 \mathrm{GHz}$ for radio communications, since these frequency bands have not yet been allocated to active services in the world.

\section{General consideration and expectations of THz waves for communications}

From Shannon formula ${ }^{11}$, the information capacity, $C$, or the data rate is associated to the bandwidth, $W$, and the signal-to-noise ratio, $S / N$, as is given by $C[\mathrm{bit} / \mathrm{s}]=W \log _{2}(1$ $+S / N)$. High data-rate $\mathrm{THz}$ wireless systems could be possible due to the large available bandwidth, $W$, even though the signal power, $S$, generally tends to decrease with the carrier frequency. However, one of the big obstacles in the use of $\mathrm{THz}$ waves in wireless communications is the atmospheric attenuation ${ }^{12}$ as shown in Fig. 1a. Transmission distance is limited by the attenuation, and the appropriate carrier frequency or frequency band should be determined by applications; $100 \mathrm{GHz} \sim 150 \mathrm{GHz}$ for long distance (1 km 10 $\mathrm{km}),<350 \mathrm{GHz}$ for medium distance $(100 \mathrm{~m} \sim 1 \mathrm{~km}),<500 \mathrm{GHz}$ for in-door $(10 \sim 100 \mathrm{~m})$. Above $600 \mathrm{GHz}$, there are two windows also for in-door communications; $625 \mathrm{GHz} 725$ $\mathrm{GHz}$, and $780 \mathrm{GHz} 910 \mathrm{GHz}$. When the frequency exceeds $1 \mathrm{THz}$, the radio wave undergoes a significant absorption by water vapour and oxygen molecules in the atmosphere, and is attenuated by less than one tenth at only 1-m propagation distance, which is still useful for near-field communications (NFCs; $<0.1 \mathrm{~m}$ ). In addition, one cannot ignore attenuation from rainfall ${ }^{13}$. This attenuation is mostly independent on frequency in the range above $100 \mathrm{GHz}$, and the attenuation is about $10 \mathrm{~dB} / \mathrm{km}$ in the case of heavy rain condition $(25 \mathrm{~mm} / \mathrm{h})$, and should be considered for outdoor applications. 
A free-space path loss (FSPL) ${ }^{14}, L_{\mathrm{B}}$, which is given by $L_{\mathrm{B}}=(4 \pi d f / c)^{2}$ with link distance, $d$, carrier frequency, $f$, and the velocity of light $c$, is physically inevitable. In the first $\mathrm{THz}$ communication window $(200 \mathrm{GHz} 320 \mathrm{GHz})$ and for up to km-range systems, the link budget is very close to the FSPL, and is not really degraded by the atmospheric contribution (Fig. 1b). For $1 \mathrm{~km}$ (usual backhaul size in cellular networks), THz system will have to deal with $140-\mathrm{dB}$ total losses at a carrier frequency of $300 \mathrm{GHz}$. High gain antenna structures have to be considered to compensate this fundamental limitation. Indeed, since the antenna gain, $G_{\mathrm{A}}$, is given by $G_{\mathrm{A}}=4 \pi A \eta(f / c)^{2}$ with antenna area, $A$, and antenna efficiency, $\eta$, the free-space path loss can be compensated by the gain of both transmitter and receiver antennas; total antenna gain in the link can easily be made more than $100 \mathrm{dBi}$ at $300 \mathrm{GHz}$, though such antennas are highly directive. Even if technology will increase in output-power capability, isotropic $\mathrm{THz}$ links may not be practically feasible. Beam steering or beam forming with phased array antennas would be useful as has already been introduced in $60-\mathrm{GHz}$ wireless technologies ${ }^{15,16}$.

Depending on the above link distance criteria, promising applications of $\mathrm{THz}$ communications include front- and back-hauling of base stations (BSs) in femto cells, wireless local area networks in smart offices, wireless personal area networks in smart homes, near-field communications (NFCs) such as kiosk downloading, wireless connections in data centers, device-to-device communications (D2D), etc. (Fig. 2a). In these applications, another key aspect is the power consumption, directly related to transmitter and receiver architectures, and strongly impacting the real $\mathrm{THz}$ link scenario (mobile user or fixed point to point). For an outdoor link, spectral efficiency has to be taken into account, both for point-to-point and earth-to-space links ${ }^{17}$ in order to limit interference with established systems ${ }^{18}$. For indoor links, where frequencies can be re-used 
over several rooms for example, simple amplitude coding/duobinary can be used not only to drastically simplify the receiver architectures using primitive direct detection but also to limit the receiver energy requirements.

One of the frequently raised concerns for $\mathrm{THz}$ communications is the comparison to free-space optics (FSO) communications using infrared light (IR) waves. As we described in the beginning of the section, it would be logical to multiply the carrier frequency by several order of magnitudes and use optics as the carrier ${ }^{19-21}$. This would definitely offer more bandwidth while using the same base technology as photonics-based $\mathrm{THz}$ for the modulation of signals. However, the FSO faces lower tolerance in alignment, and requires stronger beam-steering control systems, though the development of MIMO-based system is promising ${ }^{22}$. For outdoor applications, the optics suffers more than 2 orders of magnitude higher losses in foggy conditions than $\mathrm{THz}$ waves ${ }^{23}$, which again would limit the use of the FSO systems ${ }^{24}$. Ultimately, cost, size, performance and usability would determine which becomes more adopted in the marketplace.

\section{Photonics-based approaches to realizing THz communications}

From the various $\mathrm{THz}$ technologies continuously developed, system-level efforts have led to the use of both photonics and/or electronics-based technologies. First key advances have been realized using III/V semiconductor technologies at a "lab" integration level, where each device or component separately developed is optimized to show the highest performance. Among this scheme, the highest data rates have been reached using photonic devices as transmitters, combined with cutting-edge III/V THz electronic devices as receivers. 
Photonics-based techniques offer the unique opportunity to ensure a high modulation index obtained with optical-to-THz conversion using photomixing, high-speed amplitude and/or phase coding introduced from optical coherent network technologies, which has been widely developed since 2000's and now has become a mature technology for fiber-optic core networks ${ }^{25}$. A unique feature of using photonic devices relies on the possibility to address multi-carrier transmission very easily by adding optical laser lines to the optical driving signals. In the THz range, photonic III/V (InP) devices have been led by uni-travelling carrier photodiodes (UTC-PDs) pushed up to the order of $\mathrm{mW}$ power levels around $300 \mathrm{GHz}$ in several laboratories ${ }^{26,27}$, paving the way for the realization of first indoor $\mathrm{THz}$ radio links with 'optical' data rates: for example, $50 \mathrm{Gbit} / \mathrm{s}$ at $300 \mathrm{GHz}$ using real-time amplitude signaling ${ }^{28}, 60 \mathrm{Gbit} / \mathrm{s}$ at $200 \mathrm{GHz}^{29}, 46 \mathrm{Gbit} / \mathrm{s}$ at $400 \mathrm{GHz}^{30}$ and up to $100 \mathrm{Gbit} / \mathrm{s}$ in the $100 \mathrm{GHz}$ band $^{31}$ using digital signal processing and complete solid-state receiver with active elements working at such carrier frequencies ${ }^{32}$. The way towards 100 Gbit/s systems is now paved, and future developments towards this target will drive the $\mathrm{THz}$ communication systems developments. To tackle the power limitation of photonic devices, the future of photonics-based $\mathrm{THz}$ systems may be based on the combination of power amplifiers associated to photomixers, but performances would require a monolithic association of the two devices, which has not been achieved in the $\mathrm{THz}$ range. Also, $\mathrm{THz}$ links development may also benefit of the large developments of silicon ( $\mathrm{Si}$ ) photonicsbased systems, for example at $180 \mathrm{GHz}$ a Ge-based photomixer in Si photonics process have shown EIRP (effective isotropic radiated power) $>-15 \mathrm{dBm}$ from $170 \mathrm{GHz} 190$ $\mathrm{GHz}^{33}$.

Since the early ages of $\mathrm{THz}$ communication, photonics has played a key role as a 'technology driver' for the THz transmitter (Tx). At the reception side, electronics-based 
approaches remain more efficient to achieve usable receivers (Rx's). Among THz receivers, several options have been tested for data wireless links in $\mathrm{THz}$ regime. The most common is a waveguide-integrated detector using GaAs Schottky barrier diodes (SBDs) ${ }^{34}$ initially developed for radio-astronomy applications. Harmonic mixers using GaAs SBDs have also been investigated, featuring not only efficient real-time amplitude signaling ${ }^{35}$ but also efficient down-conversion from high level THz modulation to microwave/MMW domain $^{36}$, opening the way to 'coherent fiber networks to THz radio bridges' which could be used in a future network convergence (fiber and ultra-high speed radio), as described later.

In the perspective of photonics-based $\mathrm{THz}$ transmitters for spectrally-efficient data links, the optical feed or source is requiring special attention ${ }^{28}$. Indeed, the spectral content of $\mathrm{THz}$ signal is directly related to the two optical laser lines driving photomixing devices such as photodiodes. Ultimate performance would require the optical feed featuring low jitter and narrow linewidth in the optical domain. Practically, at least the spectral separation between two laser lines have to be locked, in other words, optical laser lines should be correlated each-other to ensure a low phase noise and a limited frequency drift of the beating frequency used as a carrier in the THz domain. Several techniques have been demonstrated to achieve spectrally narrow photomixing based on laser heterodyning: for example, microchip ${ }^{37}$ or integrated lasers ${ }^{28}$, independent lasers with frequency stabilization $^{38}$ dual mode lasers ${ }^{39-41}$, Brillouin fiber lasers ${ }^{42,43}$ comb generation of a single laser line and active phase stabilization ${ }^{44}$ or III-V on Silicon dual mode lasers ${ }^{45}$.

Some features can be highlighted in these systems for $\mathrm{THz}$ communications applications: first, the use of one single laser line, modulated at $f_{0}$ (from a microwave reference) to create an optical frequency comb, further filtered using optical components to 
get $N f_{0}$ at a carrier frequency, requires an active phase stabilization to tackle random phase drifts in fiber cables. This kind of scheme has already been used to achieve real-time performance at $100 \mathrm{GHz}^{44}, 200 \mathrm{GHz}^{35}$ and $300 \mathrm{GHz}^{28}$. As the frequency increases, the frequency comb technique is limited by the increasing number of teeth to be generated in continuous wave regime (rather than common pulsed regime). In this case, a high modulating power to generate the required number of teeth and/or the use of highly nonlinear optical modulators is mandatory.

Other techniques use dual-frequency tunable lasers producing the two required lines by design (i.e., without the optical frequency comb), with tunable spectral separation from microwave ${ }^{39}$ up to sub- $\mathrm{THz}^{41}$ or $\mathrm{THz}^{40}$ frequencies. In these techniques, the common mode noise is decreased as two laser modes experience a single cavity and the spectral content is relatively invariant with frequency. Thus, the same phase noise performance (free running) can be obtained from $100 \mathrm{GHz}$ to beyond $1 \mathrm{THz}$ which is a major advantage of photonics. In ref. 40, the tuning from $\mathrm{DC}$ to $900 \mathrm{GHz}$ is achieved by the use of an electro-optic effect inside cavity. This electro-optic effect can also be used to frequency lock the THz emission thanks to an external phase locked loop, fed by the down-converted signal of a $\mathrm{THz}$ heterodyne detection. Thus, the phase noise achieved is also limited by the multiplied electrical reference used to down convert the $\mathrm{THz}$ wave. Moreover, in order to reduce the constraints on the phase locking circuits, fiber lasers can be considered in order to reduce natural optical linewidth of the free-running optical source (usually $\mathrm{MHz}$ performance from standard lasers used in optical communications). For example, Brillouin fiber lasers ${ }^{46}$ have been shown to reduce the intrinsic linewidth of an optical feed, down to $\mathrm{kHz}$ level ${ }^{43}$ around $300 \mathrm{GHz}$ and below $100 \mathrm{~Hz}$ around $1 \mathrm{THz}^{47}$. 


\section{Trends in all electronics-based approaches}

From electronic side, beyond the use of electronic receivers combined with photonic Tx, first full electronic demonstrations have been realized using standard waveguide devices that were initially developed in GaAs technology for radio-astronomy (Herschel, ALMA programs). Using commercially available sources (multiplier chains), mixers, detectors, researchers have experienced first 'lab level' THz links, in many configurations: direct amplitude modulation of the source input ${ }^{48}$ which is very simple but bandwidth limited and not suitable for phase coding of the $\mathrm{THz}$ beam as the frequency multiplication is essentially nonlinear. Using harmonic mixers as up-converters, linear behavior can be obtained and first complex signals have been transmitted using $\mathrm{THz}$ range ${ }^{49}$. In those systems, the major limitations in Tx and Rx are: (i) the nonlinear behavior of multiplier chains which limits to amplitude coding at input, (ii) the limited modulation index if the modulation is realized with a sub-harmonic mixer at source output and (iii) the relative high impedance $(\sim \mathrm{k} \Omega)$ of Schottky barrier diode-based direct detectors that limit achievable bandwidths, even if some trans-impedance amplifiers integration can partially overcome this limitation.

From these 'first-age' systems, dedicated THz circuits or sub-systems have been developed since several years, first in III/V (for THz front ends only) but silicon developments will certainly compete III/V very fast as featuring higher integration level (front-end, baseband, digital). As first demonstrations use only $\mathrm{THz}$ front ends and $\mathrm{Si}$ devices currently not fully available in THz range, and III/V have driven the first dedicated circuit-level demonstrations. For example, at reception side, fully-integrated $300-\mathrm{GHz}$ receiver MMIC using InP $\mathrm{HEMT}^{50}$, a complete I/Q receiver at $237.5 \mathrm{GHz}^{32}$ using GaAs HEMT technology, these systems being evaluated using photonics at Tx. Fully-integrated QPSK emitter and receiver chipsets using InP HEMT at $300 \mathrm{GHz}$ have also now been 
successfully validated on-wafer ${ }^{51}$ for up to $50 \mathrm{Gbit} / \mathrm{s}$ phase modulated signals, opening the way to future systems. Recently, full waveguide integrated MMIC chipset based on GaAs metamorphic HEMT at $300 \mathrm{GHz}$ have been proposed ${ }^{52}$ to handle up to $64 \mathrm{Gbit} / \mathrm{s}$ (off-line detection) in QPSK signaling over meter ranges. Fully-integrated modulation and demodulation circuits have also been achieved using DHBT in "D band" (110 GHz 170 $\mathrm{GHz})^{53}$.

Up to now, at frequencies above $100 \mathrm{GHz}, \mathrm{GaAs}$ and InP ICs have been key players in all-electronic $\mathrm{THz}$ communications research. This is mainly due to the high cutoff and maximum frequencies of transistors (for example, $\mathrm{f}_{\mathrm{t}}$ and $\mathrm{f}_{\max }$ of the order of 400 500 GHz/1000 GHz with InP DHBT ${ }^{54}$ and $660 \mathrm{GHz} />1 \mathrm{THz}$ with 20-nm mHEMT ${ }^{55}$ ). However, other technologies are also expected to open the way to practical $\mathrm{THz}$ communications, and mass production compatible chipsets are now required and rapidly developing. Si IC technologies have started to show their THz potential in the last 2-3 years. For example, SiGe HBTs are now expected to reach $700-\mathrm{GHz}$ cut-off frequencies ${ }^{56}$, and first chipsets have been already achieved: at $160 \mathrm{GHz}$ with $10-\mathrm{mW}$ power levels (1-W total consumption) and at $240 \mathrm{GHz}^{57}$, featuring output powers beyond $100 \mu \mathrm{W}, 20-\mathrm{GHz}$ bandwidth for Tx and 10-dB conversion gain for Rx. First simple tests have been achieved in free space using planar antennas and silicon lenses, over $0.3 \mathrm{~m}^{56}$. Also, wireless links using Si-CMOS are also now reported: for example, full demonstration of 11-Gbit/s links over $3 \mathrm{~m}$ have been reported at $130 \mathrm{GHz}$ with real-time performance ${ }^{58}$. All of these potential technologies for $\mathrm{THz}$ communication systems will participate to the development of the field. According to the International Technology Roadmap for Semiconductors (ITRS), the half pitch of the wiring in Si-LSIs is expected to become $10 \sim 12 \mathrm{~nm}$ by 2020 , enabling the maximum operation frequency of the mass-production level of Si-CMOS 
devices reach $1 \mathrm{THz}$, as well as various RF ICs in excess of 300-GHz operation could be realized in Si-CMOS. GaN and InP ICs, however, have the ability to significantly surpass Si devices in terms of the break-down voltage, and are still indispensable in applications where a high output power is required. A power combining technique using integrated array antennas has proven to be effective to increase an output power in Si-CMOS transmitter ICs ${ }^{59,60}$. Ultimate THz ICs would be a fusion of compound semiconductor and Si semiconductor ICs.

\section{THz link demonstrations and photonics in network convergence}

Considering all the aforementioned technologies, one can separate the types of systems in two categories; 'real time' systems, and systems with post processing. The first category, real-time systems, considers essentially a 100-\% time-availability (no latency) as the signal does not require any signal processing or off-line techniques to be analyzed. In that case, the real-time bit error rate (BER) is the usual figure of merit of the link. These 'real-time' systems have been achieved with direct detection or coherent detection (Tx and Rx have the same phase reference). As for the second category, amplitude coding, or multi-level modulation schemes (amplitude and phase coding) can also be combined with a wideband heterodyne receiver. Using further baseband of RF digital signal processing ('off-line detection'), the lack of active locking between $\mathrm{THz}$ emission and reception can be tackled, at the price of time latency. However, these 'off-line' techniques and systems have enable researchers to experience $\mathrm{THz}$ propagation of complex $\mathrm{THz}$ signals and lead to the first advanced multi-level format links. In a general data-link perspective, it contributes to increasing the knowledge in the research field, and future developments may focus on realtime systems for practical/usable systems. This 'first-age' of THz communication links can be summarized in Table $1^{61-65}$. 
One of the key features enabled by $\mathrm{THz}$ photonics technologies is the possibility to take benefit of the very low losses of optical fibers in order to remotely feed the $\mathrm{THz}$ emission circuits, which is useful in the case of backhaul applications as shown in Figs. 2a and $2 \mathrm{~b}$. Other key advantage of photonic-based solutions is the amazing facility to handle multi-carrier and multi-format THz channels as well as carrier switching (Fig. 2c), which has no equivalence in electronics-based solutions. This unique feature of photonics-based transceivers is in phase with optical network evolution towards 'flexgrids ${ }^{96,67}$ that will expand core networks bandwidth beyond traditional WDM (wavelength division multiplex) systems. By essence, photonics could play a major as a convenient 'optical-tohigh speed radio' interface in mixed network technologies context.

Figure 3 shows examples of THz link demonstrators based on photonics-based transmitters. 32-Gbit/s data transmission with 16-QAM modulation has been demonstrated with a link distance of $25 \mathrm{~m}$ at a carrier frequency of $385 \mathrm{GHz}$ using a setup of Fig. 3a, while 50-Gbit/s real-time transmission with OOK modulation has been performed with a link distance of $100 \mathrm{~m}$ at a carrier frequency of $330 \mathrm{GHz}$ using a setup of Fig. 3b. In the latter case, reflector antennas were used to realize a gain of over $53 \mathrm{dBi}$.

\section{Future prospect and challenges}

The full deployment of a THz-based wireless communication technology is facing multiple challenges. As discussed in the previous section while photonic technologies can help in term of link efficiency, generating high data rate and coherence, the system still needs more output power at the transmitter in particular for applications such as backhaul where the distance will have to reach a kilometre. For example, a state of the art photonic emitter will offer about $1-\mathrm{mW}$ output power at $300 \mathrm{GHz}^{26}$ and easily $40-\mathrm{Gbit} / \mathrm{s}$ data rate in a $50-$ 
GHz DSB bandwidth, while a state of the art room temperature operating receiver (Schottky-diode mixer based ${ }^{68}$ ) would offer a detection sensitivity of $4 \cdot 10^{-19} \mathrm{~W} / \mathrm{Hz}$. With such characteristics and 40-dBi gain antennas, the maximum distance achievable in a worst case scenario $\left(10-\mathrm{dB} / \mathrm{km}^{69}\right.$ attenuation for heavy rain) would be $280 \mathrm{~m}$; short of the needed $1 \mathrm{~km}$. While modulating in optics could easily reach very high data rate (for example $100 \mathrm{Gbit} / \mathrm{s}^{70}$ ) making the modulator a lower priority, it is clear that a better transmitter and/or receiver are required together with higher antenna gains. Another challenge is related to energy consumption as data traffic especially the wireless part of the traffic will soon be the highest consumption of energy per habitant in the world ${ }^{71}$. Such challenges will still require developments of technologies to generate more power at the transmitter while increasing the overall system efficiency. In this section, we are discussing the different prospects that could potentially fully enable the future THz wireless network, as summarized in Table 2.

The first technology that could improve both the power at the transmitter and the overall efficiency of the system is 'photonic integration'. Photonic integration will naturally reduce coupling losses, such as the loss from fibre to chip and in particular the loss between the laser and the photomixer. It should also enable the use of multiple antenna system that would lead to advanced active array antennas to compensate the path loss and allow for some tracking. It is interesting to see that photonic integration is now progressing fast in the world with a multi-wafer foundry platform system in Europe ${ }^{72}$ and a large research investment in American institutes for manufacturing integrated photonics ${ }^{73}$. These have led to important progresses for the field of $\mathrm{THz}$ communications with highlights such as the development of a single chip transmitter (Fig. 4a) emitting $100 \mu \mathrm{W}$ at $100 \mathrm{GHz}$ from a total electrical consumption of $1 \mathrm{~W}$ including cooling ${ }^{74,75}$. That chip was also used 
successfully in transmission systems ${ }^{76}$. However, one can note that in such a case the power is still limited for long distance transmission, and recent development of integrated multiple photodiodes ${ }^{26}$ (Fig. 4b) is promising to overcome the limitation.

Further to these recent progress in integration the development of active $\mathrm{Si}$ photonics integrated technology ${ }^{77-80}$ would enable potentially even better efficiency, easier integration with Si electronics technology and lower loss waveguides. Interestingly, knowing that the best photomixer saturation limit is mostly thermally driven, the performances at lower frequencies of UTC integrated on Si substrates with higher saturation due to the better thermal properties of Si offers a lot of prospect ${ }^{81}$.

However, even with such developments, there is still a clear need for amplification at both the emitter and receiver, thus low-noise and wide bandwidth $\mathrm{THz}$ amplifiers for both transmitters and receivers are also a key priority. One example of such progress in amplifiers is a THz amplifiers based on InP HBTs with a record high bandwidth of 235 GHz developed by UCL with Chalmers University ${ }^{82}$. Also the hybrid/monolithic integration of UTC-PDs with HEMT/HBT amplifiers for emission at $100 \mathrm{GHz}$ seems to demonstrate an interesting potential for future components of the system ${ }^{83-85}$.

$\mathrm{THz}$ technologies could benefit further if a strong interconnect technology is created to direct the THz wave on chip between different components. For that we could highlight low loss waveguide technology, where hollow waveguide with loss lower than $0.2 \mathrm{~dB} / \mathrm{m}$ have been developed ${ }^{86}$. However, considering the architecture of a photonic chip, a planar solution would be more interesting, and developments in photonic band-gap structures that have been integrated directly with photonic chips and antenna ${ }^{87}$ (Fig. 4c) would offer great prospect. Further development in plasmonic-based waveguides ${ }^{88}$ could offer both low enough loss, field enhancement for interaction with a modulator or detection 
system and size reduction that are all desirable features in a future THz system. Further to such structures, the development in metamaterials can enable enhanced THz manipulation in devices ${ }^{89}$, in particular interesting work has been done on $\mathrm{THz}$ modulators using metamaterials $^{90,91}$.

The development of graphene-based technology for $\mathrm{THz}$ is also a promising area of development in particular for enhanced detection and emission using graphene-based field effect transistors $^{92,93}$, and modulators in graphene-based metamaterials ${ }^{94}$ (Fig. 4d), though in most cases the current performances are not at the state of the art compared to photonics-only-based technologies, the physical properties of a graphene and the level of results are indeed encouraging. In particular, the developments of room temperature detectors would be interesting in order to enhance the detection sensitivity of the system ${ }^{95}$.

Also, plasma-based transistors have been shown to be potential candidates for $\mathrm{THz}$ detecton $^{96-97}$, mainly for imaging, but have also been investigated in data communication for $300 \mathrm{GHz}$ short-range links ${ }^{98}$. The main limitation issue of these devices is the output interconnection: as the plasma is enhanced near transistor pinch-off voltage, the channel impedance value is quite high and far from usual wideband amplifier impedances $(50 \Omega)$. In the future, innovative interconnects need to be developed to take full benefit of the plasma effects.

Finally, every radio wave at frequencies below $3 \mathrm{THz}$ should be regulated and be got global consensus in its use for passive and active services. The effort on standardization and spectrum regulation issues for $\mathrm{THz}$ communications has been initiated and led by Kürner et al. ${ }^{2,18}$ by considering use cases, channel/propagation models, interference effects with other services such as, for example, radio astronomy and earth observation, enabling technologies, etc. 


\section{References}

1. Cisco; and/or its Affiliates, The Zettabyte Era: Trends and Analysis. Cisco Vis. Netw. Index, 1-24 (2014).

2. Kürner, T. \& Priebe, S., Towards THz communications - status in research, standardization and regulation. Journal of Infrared, Millimeter, and Terahertz Waves 35 (1), 53-62 (2014).

3. Bird, T. S., Recent progress in microwave antennas for wireless communications. presented at Asia-Pacific Microwave Conference (APMC2011), Keynote address, Melbourne, Australia, 2011.

4. Pagani, M., Microwave digital radio link transceivers: historical aspects and trends. presented at IEEE Intern. Microwave Symposium (IMS2015), Workshop WHM-4, Phoenix, 2015.

5. Niu, Y., Li, Y., Jin, D., Su, L. \& Vasilakos, A. V., A survey of millimeter wave communications (mmWave) for 5G: opportunities and challenges, Wireless Networks 21(8), 2657-2676 (2015).

6. Nagatsuma, T. et al., A 120-GHz Integrated photonic transmitter, presented at IEEE Topical Meeting on Microwave Photonics (MWP2000), Oxford, 2000.

7. Kleine-Ostmann, T., Pierz, K., Hein, G., Dawson, P. \& Koch, M., Audio signal transmission over $\mathrm{THz}$ communication channel using semiconductor modulator. Electronic Letters 40 (2), 124-125 (2004). 
8. Liu, T.-A., Lin, G.-R., Chang, Y.-C., \& Pan, C.-L., Wireless audio and burst communication link with directly modulated $\mathrm{THz}$ photoconductive antenna. Optics Express 13(25), 10416-23 (2005).

9. Hirata, A. et al., 120-GHz-band wireless link technologies for outdoor 10-Gbit/s data transmission. IEEE Trans. Microwave Theory and Techniques 60 (3), 881-895 (2012).

10. Takahashi, H. et al., 120-GHz-band 20-Gbit/s transmitter and receiver MMICs using quadrature phase shift keying, presented at proceedings of the 2012 7th European Microwave Integrated Circuit Conference (EuMIC), Amsterdam, 2012

11. Shannon, C. E., Communication in the presence of noise. Proceedings of the Institute of Radio Engineers 37, 10-21 (1949).

12. ITU-R P.676-6, Attenuation by atmospheric gases, 2005.

13. ITU-R P.838-3, Specific attenuation model for rain for use in prediction methods, 2005.

14. Pozar, D. M., Microwave Engineering 4-th edition, John Wiley and Sons Ltd, New York, United States, 2011.

15. http://www.sibeam.com/Products.aspx

16. Han, S., Xu, C. I, Z. \& Rowell, C., Large-scale antenna systems with hybrid analog and digital beamforming for millimeter wave 5G, IEEE Communications Magazine 53(1), 186-194 (2015).

17. Suen, J. Y., Fang, M. T., Denny, S. P. \& Lubin, P. M., Modeling of Terabit geostationary Terahertz satellite links from globally dry locations. IEEE Trans. Terahertz Science and Technology 5 (2), 299-313 (2015).

18. http://www.ieee802.org/15/pub/index_TG3d.html 
19. Tsonev, D., Videv, S. \& Haas, H., Towards a $100 \mathrm{~Gb} / \mathrm{s}$ visible light wireless access network. Optics Express 23 (2), 1627-1637 (2015).

20. Zheng, Z., Liu, L., Chen, T. \& Hu, W. W., Integrated system of free-space optical and visible light communication for indoor wireless broadband access. Electronics Letters 51 (23), 1943 (2015).

21. Oh, C. W., Tangdiongga, E. \& Koonen, A. M. J., Steerable pencil beams for multiGbps indoor optical wireless communication. Optics Letters 39 (18), 5427-5430 (2014).

22. Fath, T. \& Haas, H., Performance comparison of MIMO techniques for optical wireless communications in indoor environments. IEEE Trans. Communications 61 (2), 733-742 (2013).

23. ITU-R P.840-3, "Attenuation due to clouds and fog," 1999.

24. Ma, J, Vorrius F., Lamb L., Moeller, L., Federici J.F, Experimental comparison of terahertz and infrared signaling in laboratory-controlled rain. J. Infrared Milli Terahz Waves 36, 856-865 (2015).

25. Richardson, D. J., Fini, J. M. \& Nelson, L. E., Space-division multiplexing in optical fibres. Nature Photonics 7, 354-362 (2013).

26. Song, H. J. et al., Uni-travelling-carrier photodiode module generating $300 \mathrm{GHz}$ power greater than $1 \mathrm{~mW}$. IEEE Microwave and Wireless Components Letters 22 (7), 363-365 (2012).

27. Wun, J. M., Lai, C. H., Chen, N. W., Bowers, J. E. \& Shi, J. W., Flip-Chip Bonding Packaged THz Photodiode With Broadband High-Power Performance. IEEE Photonics Technology Letters 26 (4), 2462-2464 (2014). 
28. Nagatsuma, T. \& Carpintero, G., Recent progress and future prospect of photonicsenabled terahertz communications research. IEICE Trans. Electronics E98-C (12), 1060-1070 (2015).

29. Shams, H. et al., Photonic generation for multichannel $\mathrm{THz}$ wireless communication. Optics Express 22 (19), 23465-123472 (2014).

30. Ducournau, G. et al., Ultrawide bandwidth single channel $0.4 \mathrm{THz}$ wireless link combining broadband quasi-optic photomixer and coherent detection. IEEE Trans. Terahertz Science and Technologies 4 (3), 328-337 (2014).

31. Li, X. et al., A 400G optical wireless integration delivery system. Optics Express 21 (16), 187894-187899 (2013).

32. Koenig, S. et al., Wireless sub-THz communication system with high data rate. Nature Photonics 7 (12), 977-981 (2013).

33. Bowers, S. M., Abiri, B., Aflatouni, F. \& Hajimiri, A., A compact optically driven travelling-wave radiating source, presented at 2014 Optical Fiber Communications Conference and Exhibition (OFC), San Francisco (USA), 2014.

34. Nagatsuma, T. et al., Terahertz wireless communications based on photonics technologies. Optics Express 21 (20), 477-487 (2013).

35. Ducournau, G. et al., Coherent THz communication at $200 \mathrm{GHz}$ using a frequency comb, UTC-PD and electronic detection. Electronics Letters 50 (5), 386-388 (2014).

36. Kanno, A. et al., Coherent terahertz wireless signal transmission using advanced optical fiber communication technology. Journal of Infrared, Millimeter, and Terahertz Waves 36 (2), 180-197 (2015). 
37. Hyodo, M, Tani, M, Matsuura, S, Onodera, N and Sakai, K, Generation of millimetre-wave generation using a dual-longitudinal mode microchip laser, Electronics Letters 32 (17), 1589 (1996).

38. Cliche, J.-F., Shillue, B., Têtu, M. and Poulain, M. A 100-GHz-tunable photonic millimeter wave synthesizer for the Atacama Large Millimeter Array radiotelescope, presented at IEEE Intern. Microwave Symposium (IMS2007).

39. Pillet G. et al., Dual-frequency laser at $1.5 \mu \mathrm{m}$ for optical distribution and generation of high-purity microwave signals IEEE J. Lightwave Technology 26(15), 2764 (2008).

40. Danion G. et al., Dual frequency laser with two continuously and widely tunable frequencies for optical referencing of GHz to THz beatnotes Optics Express 22(15), 17673-17678 (2014).

41. Pillet G. et al., Dual-Frequency Laser Phase Locked at $100 \mathrm{GHz}$ IEEE J. Lightwave Technology 32(20), 3824-3830 (2014).

42. Gross, M.C. et al., Tunable millimeter-wave frequency synthesis up to $100 \mathrm{GHz}$ by dual-wavelength Brillouin fiber laser, Optics Express 18(13), 13321-13330 (2010).

43. Ducournau, G. et al., Highly coherent $\mathrm{THz}$ wave generation with a dual frequency Brillouin fiber laser and a $1.55 \mu \mathrm{m}$ photomixer, Optics Letters 36(11), 2044-2046 (2011).

44. Yoshimizu Y. et al., Wireless transmission using coherent terahertz wave with phase stabilization, IEICE Electronics Express 10(18), 578-585 (2013).

45. Shao, H. et al., Heterogeneously integrated III-V/silicon dual-mode distributed feedback laser array for THz generation Optics Letters, 39(22), 6403-6406 (2014). 
46. Debut, A., Randoux, S. and Zemmouri, J., Experimental and theoretical study of linewidth narrowing in Brillouin fiber ring lasers J. Opt. Soc. Am. B, 18(4), 556-567 (2001).

47. Ducournau G. et al., Cascaded Brillouin fibre lasers coupled to unitravelling carrier photodiodes for narrow linewidth terahertz generation. Electronics Letters, 50(9) 690692, 2014.

48. Moeller, L., Federici, J. \& Su, K., 2.5Gbit/s duobinary signaling with narrow bandwidth 0.625 terahertz source. Electronics Letters 47 (15), 856-858 (2011).

49. Jastrow, C. et al., Wireless digital data transmission at $300 \mathrm{GHz}$. Electronics Letters 46 (9), 661-663 (2010).

50. Song, H. J., Kim, J. Y., Ajito, K., Yaita, M. \& Kukutsu, N., Fully Integrated ASK receiver MMIC for terahertz communications at $300 \mathrm{GHz}$. IEEE Trans. Terahertz Science and Technology 3 (4), 445-452 (2013).

51. Song, H. J., Kim, J. Y., Ajito, K., Kukutsu, N. \& Yaita, M., 50-Gb/s direct conversion QPSK modulator and demodulator MMICs for terahertz communications at $300 \mathrm{GHz}$. IEEE Trans. Microwave Theory and Techniques 62 (3), 600-609 (2014).

52. Kallfass, I. et al., Towards MMIC-based $300 \mathrm{GHz}$ indoor wireless communication systems. IEICE Trans. Electronics E98-C (12), 1081-1088(2015).

53. Carpenter, S., Abbasi, M. \& Zirath, H., Fully Integrated D-band direct carrier quadrature (I/Q) modulator and demodulator circuits in InP DHBT technology. IEEE Trans. Microwave Theory and Techniques 63 (5), 1666-1675 (2015).

54. Zaknoune, M., III-V technologies for $\mathrm{mm} W$ and THz applications, presented at IEEE Intern. Microwave Symposium (IMS2015), Workshop WSI-2, Phoenix, 2015. 
55. Schlechtweg, M., Multifunctional circuits and modules based on III/V mHEMT technology for (Sub-)millimeter-wave applications in space, communication and sensing, presented at IEEE Intern. Microwave Symposium (IMS2015), Workshop WSI-6, 2015.

56. Pfeiffer, U., RF front-ends for mm-Wave and THz application in SiGe/CMOS, presented at IEEE Intern. Microwave Symposium (IMS2015), Workshop WSI-5, Phoenix, 2015.

57. Zhao, Y., Ojefors, E., Aufinger, K., Meister, T. F. \& Pfeiffer, U. R., A $160 \mathrm{GHz}$ subharmonic transmitter and receiver chipset in an SiGe HBT technology. IEEE Trans. Microwave Theory and Techniques 60 (10), 3286-3299 (2012).

58. Fujishima, M., Amakawa, S., Takano, K., Katayama, K. \& Yoshida, T., Terahertz CMOS design for low-power and high-speed wireless communication. IEICE Trans. Electronics E98-C (12) 1091-1104(2015).

59. Sengupta, K. \& Hajimiri, A., A $0.28 \mathrm{THz}$ power-generation and beam-steering array in CMOS based on distributed active radiators. IEEE J. Solid-State Circuits 47(12), 3013-3031 (2012).

60. Han, R. \& Afshari, E., A CMOS high-power broadband 260-GHz radiator array for spectroscopy. IEEE J. Solid-State Circuits 48(12), 3090-3104 (2013).

61. Kallfass, I. et al., $64 \mathrm{Gbit} / \mathrm{s}$ transmission over $850 \mathrm{~m}$ fixed wireless link at $240 \mathrm{GHz}$ carrier frequency, Journal of Infrared Milli. Terahz Waves 36, 221-233 (2015).

62. Ducournau G. et al., $32 \mathrm{Gbit} / \mathrm{s}$ QPSK transmission at $385 \mathrm{GHz}$ using coherent fibreoptic technologies and $\mathrm{THz}$ double heterodyne detection. Electronics Lett. 12(11), 915-917 (2015). 
63. Yu X. et al., $60 \mathrm{Gbit} / \mathrm{s} 400 \mathrm{GHz}$ wireless transmission. presented at Intern. Conference on Photonics in Switching (PS2015), Florence Italy, 2015.

64. Moeller L., Federici J. \& Su. K., 2.5Gbit/s duobinary signalling with narrow bandwidth 0.625 terahertz source. Electronics Lett. 47(15), 856-858, (2011).

65. Wang C. et al., 0.34-THz wireless link based on high-order modulation for future wireless local area network applications. IEEE Trans. Terahertz Science and Technology, 4(1) 75-85 (2014).

66. http://www.lightwaveonline.com/articles/print/volume-30/issue2/Features/superchannels-flex-grid-multilayer-switching.html.

67. Winzer, P.J. \& Essiambre, R., Advanced optical modulation formats. Proceedings of the IEEE, 94(5), 952-985 (2006).

68. Crowe, T. W. et al., GaAs Schottky diodes for THz mixing applications, Proceedings of the IEEE 80 (11), 1827-1841 (1992).

69. Nicholas, C. E. B., Currie, C., Principles and Applications of Millimetre Wave Radar. Norwood, MA: Artech House, 1987.

70. Maher, R., Alvarado, A., Lavery, D. \& Bayvel, P., Increasing the information rates of optical communications via coded modulation: a study of transceiver performance, Scientific Reports 6, 21278 (2016).

71. Martiskainen, M. \& Coburn, J., The role of information and communication technologies (ICTs) in household energy consumption-prospects for the UK. Energy Efficiency 4 (2), 209-221 (2011).

72. Smit, M. et al., Generic foundry model for InP-based photonics. IET Optoelectronics $\mathbf{5}$ (5), 187-194 (2011).

73. http://www.aimphotonics.com/. 
74. Balakier, K. et al., Optical injection locking of monolithically integrated photonic source for generation of high purity signals above $100 \mathrm{GHz}$. Optics Express 22 (24), 29404-29412 (2014).

75. van Dijk, F. et al., Integrated InP heterodyne millimeter wave transmitter. IEEE Photonics Technology Letters 26 (10), 965-968 (2014).

76. Carpintero, G. et al., Microwave photonic integrated circuits for millimeter-wave wireless communications. IEEE J. Lightwave Technology 32 (20), 3495-3501 (2014).

77. Heck, M. J. R. et al., Hybrid silicon photonic integrated circuit technology. IEEE J. Selected Topics in Quantum Electronics 19 (4) (2013).

78. Baets, R. et al., Integration of photonic functions in and with silicon, presented at the 34th European Solid-State Device Research Conference (ESSDERC2004), Leuven Belgium, 2004.

79. Duan, G. H. et al., Hybrid III-V on silicon lasers for photonic integrated circuits on silicon. IEEE J. Selected Topics in Quantum Electronics 20 (4), 6100213 (2014).

80. Lee, A. D. et al., InAs/GaAs Quantum-dot lasers monolithically grown on $\mathrm{Si}, \mathrm{Ge}$, and Ge-on-Si substrates. IEEE J. Selected Topics in Quantum Electronics 19 (4) (2013).

81. Beling, A. et al., InP-based waveguide photodiodes heterogeneously integrated on silicon-on-insulator for photonic microwave generation. Optics Express 21 (22), 25901-25906 (2013).

82. Eriksson, K., Darwazeh, I. \& Zirath, H., InP DHBT distributed amplifiers with up to 235-GHz bandwidth. IEEE Trans. Microwave Theory and Techniques 63 (4), 1334$1341(2015)$. 
83. Umezawa, T. et al., High conversion gain, low power consumption W-band photoreceiver integrated with UTC-PD and InP-PHEMT amplifier, presented at IEEE Topical Meeting in Microwave Photonics, Paphos Cyprus, 2015.

84. Ito, H., Furuta, T., Hirata, A., Kosugi, T., Muramoto, Y., Tokumitsu, M. Nagatsuma, T. \& Ishibashi, T., Pre-amplifier integrated uni-travellingcarrier photodiode module for operation in $120 \mathrm{GHz}$ band, Electron. Lett. 41(6), 360-362 (2005).

85. Kashio, N., Kurishima, K., Sano K., \& Ida, M., Watanabe N. \& Fukuyama, H., Monolithic integration of InP HBTs and uni-traveling-carrier photodiodes using nonselective regrowth, IEEE Transactions on Electron Devices 54(7), 1651-1657 (2007).

86. Mitrofanov, O., Tan, T., Mark, P. R., Bowden, B. \& Harrington, J. A., Waveguide mode imaging and dispersion analysis with terahertz near-field microscopy. Applied Physics Letters 94 (17), 171104-1-3 (2009).

87. Tsuruda, K., Fujita \& Nagatsuma, T., Extremely low-loss terahertz waveguide based on silicon photonic-crystal slab. Optics Express 23 (25), 250727 (2015).

88. Zhan, H., Mendis, R. \& Mittleman, D. M., Superfocusing terahertz waves below lambda/250 using plasmonic parallel-plate waveguides. Optics Express 18 (9), 96439650 (2010).

89. Withayachumnankul, W. \& Abbott, D., Metamaterials in the terahertz regime. IEEE Photonics Journal 1 (2), 99-118 (2009).

90. Savo, S., Shrekenhamer, D. \& Padilla W. J., Liquid Crystal Metamaterial Absorber Spatial Light Modulator for THz Applications. Advanced Optical Materials 2 (3), 275279 (2014). 
91. Zhang, Y. et al., Gbps terahertz external modulator based on a composite metamaterial with a double-channel heterostructure. Nano Letters 15 (5), 3501-3506 (2015).

92. Abbas, A., Karabiyik, M. \& Pala, N., Graphene-based field-effect transistor structures for terahertz applications, presented at Terahertz Physics, Devices, and Systems Vi: Advanced Applications in Industry and Defense, 2012.

93. Tong, J. Y., Muthee, M., Chen, S. Y., Yngvesson, S. K. \& Yan, J., Antenna Enhanced graphene THz emitter and detector. Nano Letters 15 (8), 5295-5301 (2015).

94. Luxmoore, I. J. et al., Highly efficient modulation of THz metamaterials using graphene surface plasmons, presented at Conference on Lasers and Electro-Optics (CLEO2015), San Jose, 2015.

95. Mittendorff, M. et al., Ultrafast graphene-based broadband THz detector, Applied Phys. Lett. 103, 021113 (2013).

96. Knap, W. et al., Resonant detection of subterahertz radiation by plasma waves in a submicron field-effect transistor, Applied Physics Letters 80(18) 3433-3435 (2010).

97. Otsuji, T., Trends in the research of modern terahertz detectors: plasmon detectors, IEEE Trans. Terahertz Science and Technology, 5(1) 1110-1120 (2015).

98. Tohme, L. et al., Terahertz wireless communication using GaAs transistors as detectors, Electron. Lett. 50(4) 323-325 (2014).

\section{Acknowledgements}

T. N. acknowledges the Ministry of Internal Affairs and Communications (MIC) Japan for funding the Strategic Information and Communications R\&D Promotion Programme (SCOPE), and Japan Science and Technology Agency (JST) for funding Industry-Academia 
Collaborative R\&D Program. G. D. gratefully acknowledges the French Agence Nationale de la Recherche (ANR) for funding the COM'TONIQ 'Infra' 2013 program on THz communications, through the grant ANR-13-INFR-0011-01, and the support from several French research programs and institutes: Lille University, IEMN institute (RF/MEMS characterization center, nanofab and Telecom platforms, IRCICA), the CNRS, and the Renatech nano fabrication network in France. This work was also partly supported by the French “Programmes d'investissement d'avenir" Equipex FLUX 0017, ExCELSiOR project and the Nord-Pas de Calais Regional council and the FEDER through the CPER 'Photonics for Society'. C. R. acknowledges the UK Engineering and Physical Science Research Council for its funding of the programme grant on Coherent Terahertz systems and the European commission for its support of the IPHOBAC-NG project and FiWin5G Marie Curie ITN. 


\section{Figure/table legends}

Figure 1 | Impact of atmospheric attenuation of THz waves. a, Transmission distance is limited by the attenuation, and the appropriate carrier frequency or frequency band is chosen by applications such as long distance, medium distance, in-door, and nearfield communications (NFCs). Above $600 \mathrm{GHz}$, there are two windows for in-door communications; $625 \mathrm{GHz} 725 \mathrm{GHz}$, and $780 \mathrm{GHz} \sim 910 \mathrm{GHz}$. b, Link budget at $\mathrm{THz}$ frequencies for the isotropic case ( $\mathrm{Tx}$ and $\mathrm{Rx}$ antennas with 0 - $\mathrm{dBi}$ gain) for $23^{\circ} \mathrm{C}$ and $2.59 \%$ water content in air composition (tropical climate).

Figure 2 | Application of THz link in networks. a, Prospective view of THz link to connect base stations (BSs) for backhauling as well as device-to-device (D2D) interface. $\mathbf{b}$, Concept of fiber-to-THz radio bridges for seamless connection between fiber-optic link and $\mathrm{THz}$ link ensuring the same data modulation formats with no latency. c, Configuration of multi-carrier reconfigurable and frequency-agile THz links using photonics.

Figure 3 | Examples of THz links using photonics-based transmitters. a, multilevel 32-Gbit/s link over $25 \mathrm{~m}$ with 16-QAM modulation delivered by fiber-optic network. b, Real-time 50-Gbit/s link over 100 m with OOK modulation.

Figure 4 | Enabling technologies for future THz communications. a, Integrated photonic transmitter at $100 \mathrm{GHz}^{75}$. b. Integrated photodetectors with $1 \mathrm{~mW}$ output at 300 $\mathrm{GHz}^{26}$. c, Photonic band-gap $\mathrm{THz}$ waveguides for interconnects ${ }^{87}$. d, Graphene-based, metamaterial structured THz modulator ${ }^{94}$. 
Table 1 Reported THz systems and actual highest performances achieved using several technologies. CDP is the capacity $\times$ distance product, in Gbit/s.km. CDP is a figure of merit for communication systems assuming the maximal regeneration-free distance in real-time conditions $^{67}$. Most of highest data-rate of $\mathrm{THz}$ wireless systems have been achieved using the $\mathrm{THz}$ photonics technologies at transmitter, mainly based on high speed photodiodes. Combination of polarization, frequencies are now investigated to increase data-rate in the available THz bandwidth. SHM: Sub-Harmonic Mixer, UTC: Uni-Travelling Carrier, HEMT: High Electron Mobility Transistor, MMIC: Monolithic Microwave Integrated Circuit, SBD: Schottky Barrier Diode, ASK: Amplitude Shift Keying, QPSK: Quadrature Shift Keying, QAM: Quadrature Amplitude Modulation.

Table 2 Summary of requirement and challenges for future $\mathrm{THz}$ communications technologies. 

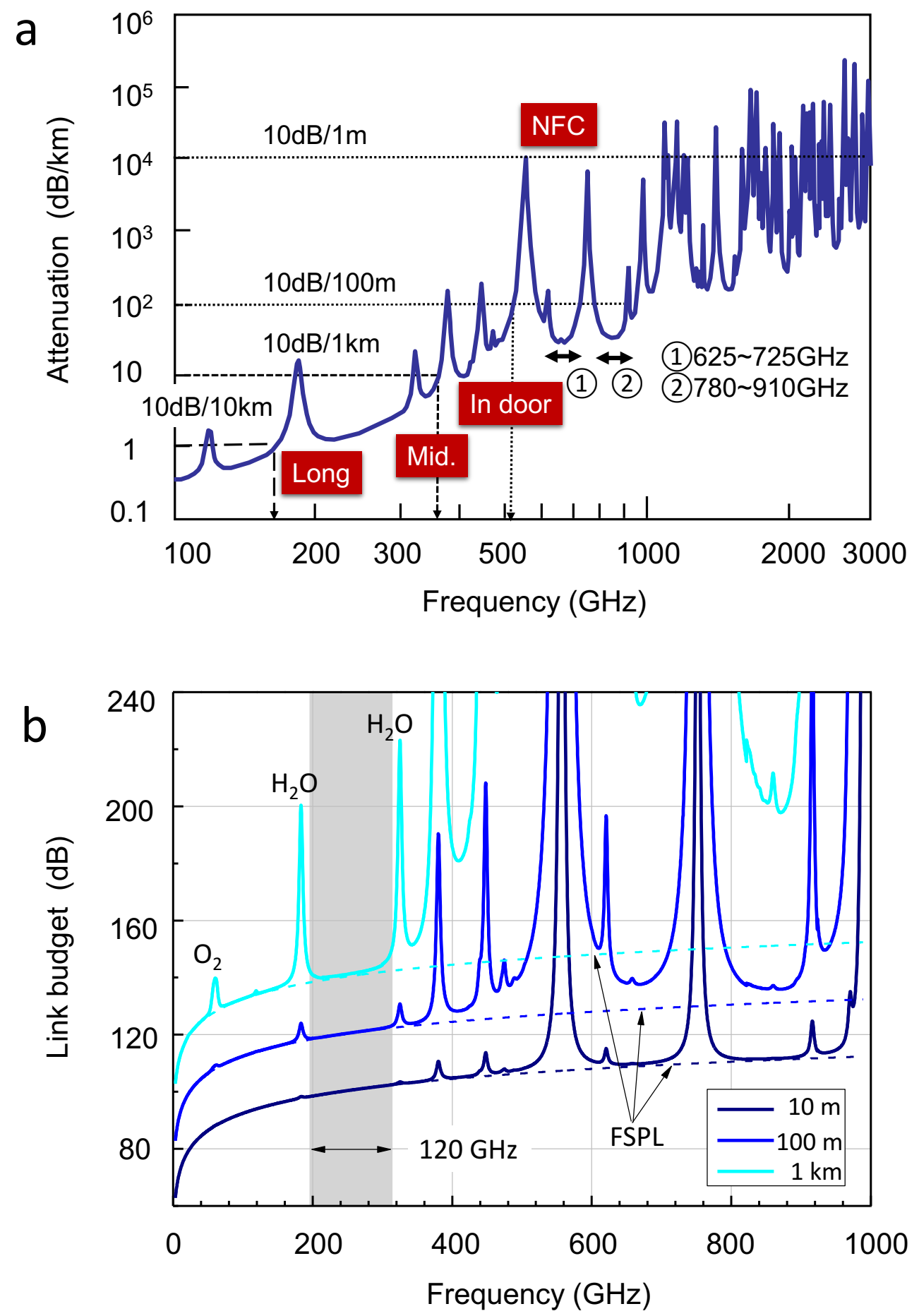

Figure 1 
a

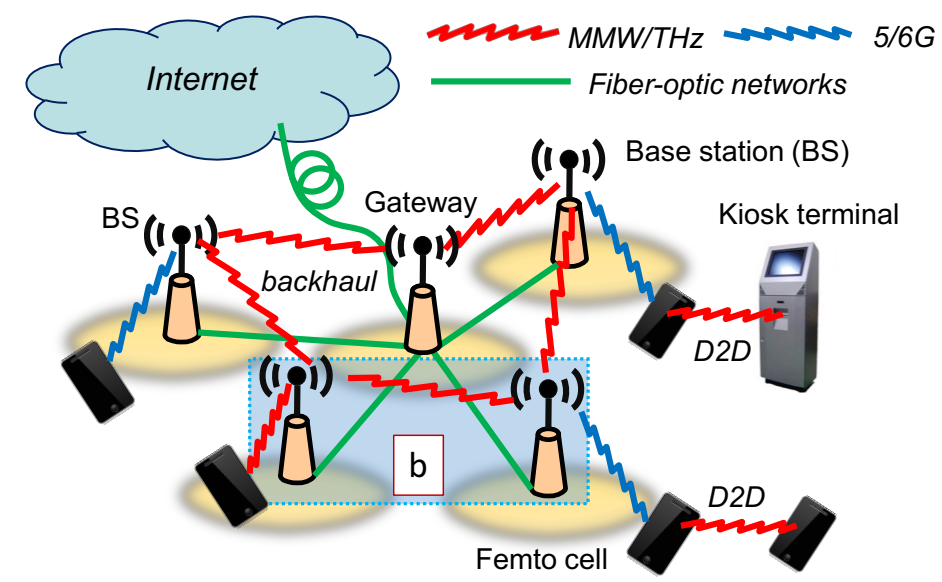

b

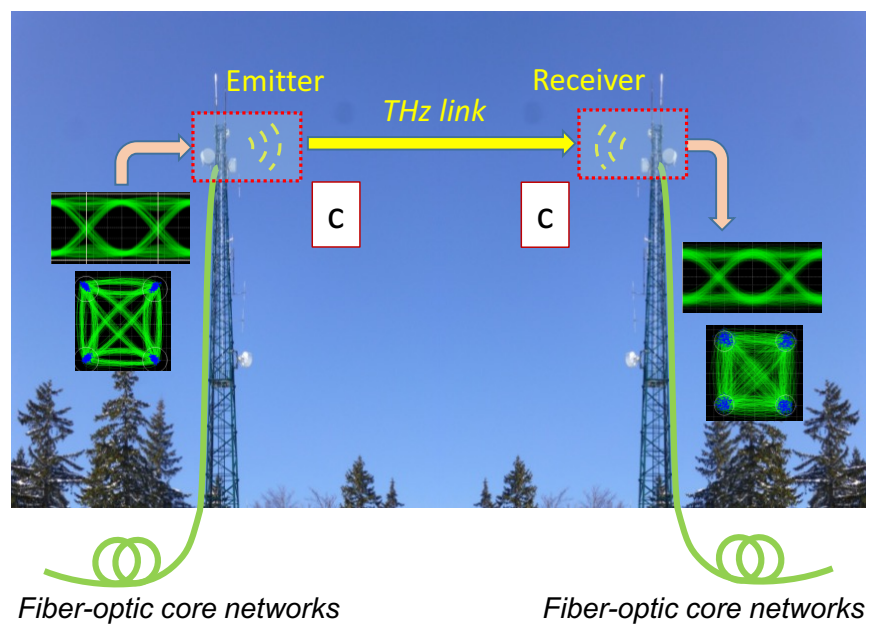

C

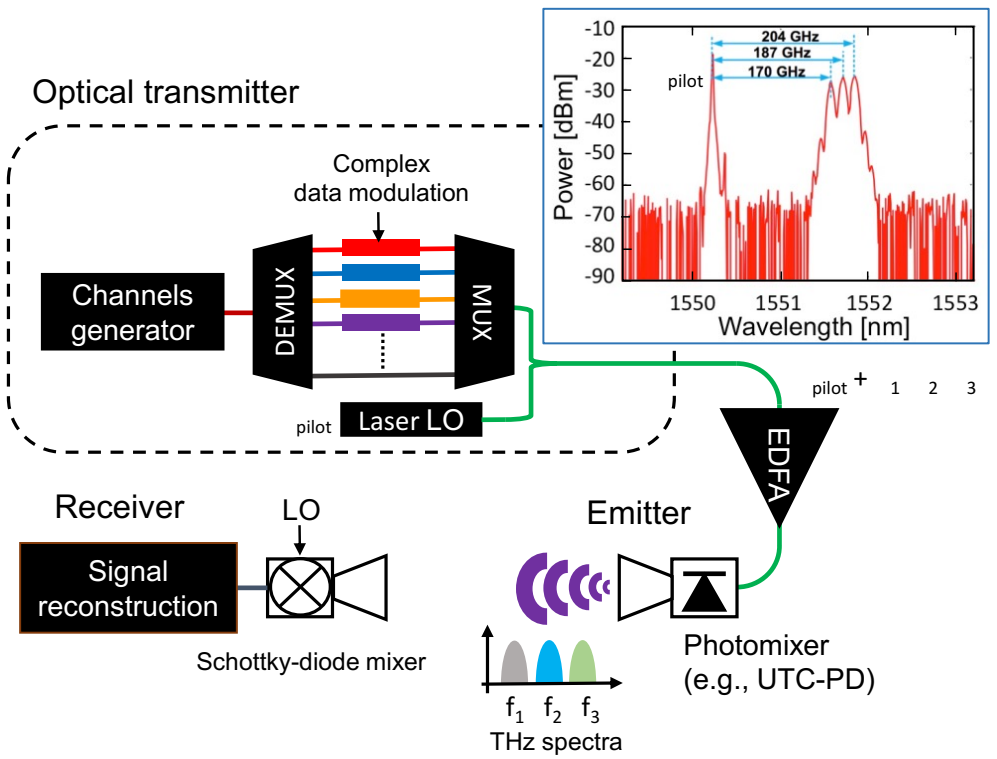

Figure 2 

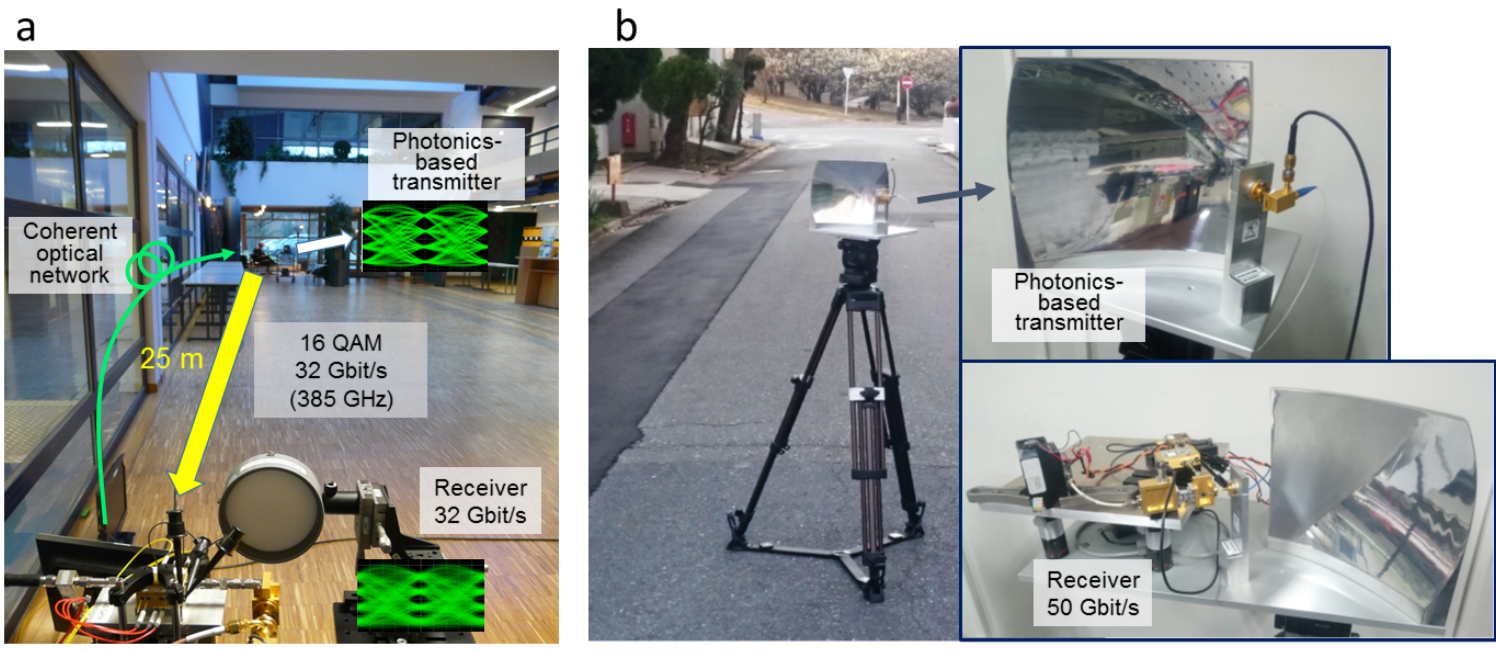

Figure 3 


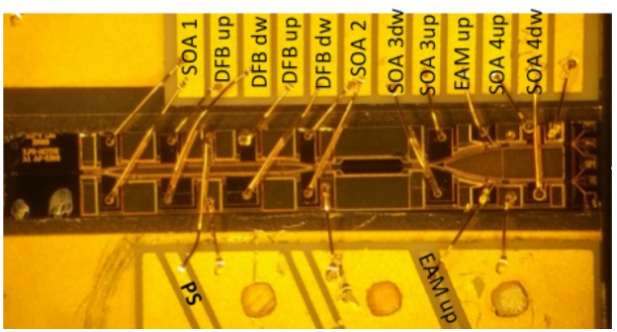

WR-3 Hollow metallic

C

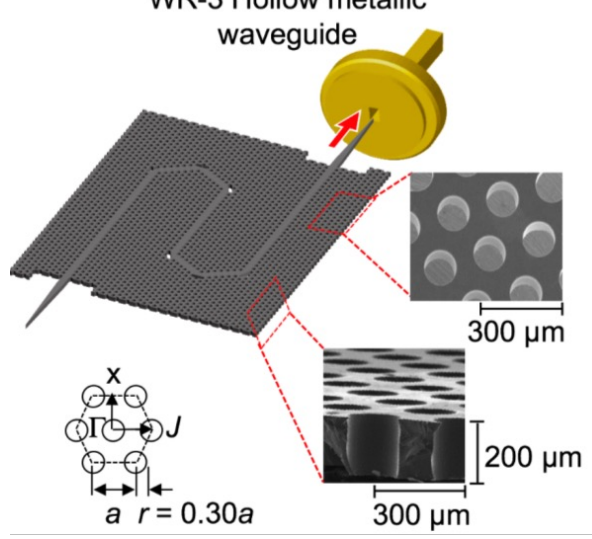

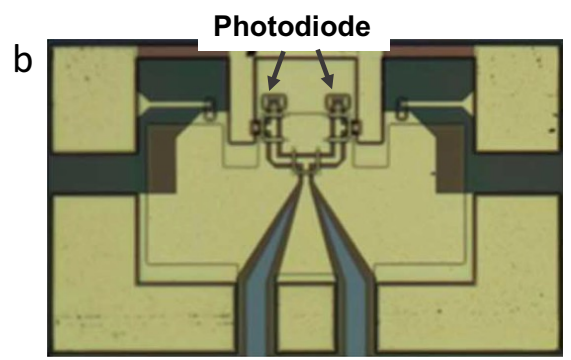

d

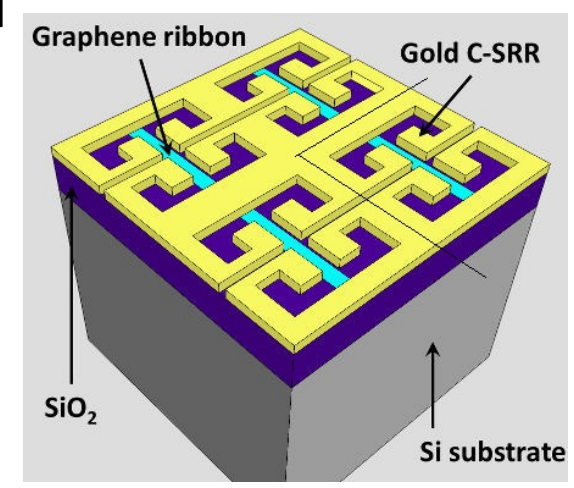

Figure 4 


\section{Table 1}

\begin{tabular}{|c|c|c|c|c|c|c|c|c|c|}
\hline $\begin{array}{c}\text { Data rate } \\
\text { (Gbit/s) }\end{array}$ & $\begin{array}{l}\text { Distance } \\
\text { (m) }\end{array}$ & $\begin{array}{l}\text { Freq. } \\
\text { (GHz) }\end{array}$ & Multiplexing & $\begin{array}{l}\text { Technology } \\
\text { (Tx/Rx) }\end{array}$ & Modulation & BER (Type) & Ref. & $\begin{array}{c}\text { CDP } \\
\text { (Gbit/s.km) }\end{array}$ & Year \\
\hline 200 & 0.5 & 100 & Polarization (2 ch) & PD / SHM & QPSK & $10^{-3} /$ off-line & [31] & - & 2013 \\
\hline 10 & 1000 & 120 & - & $\begin{array}{c}\text { UTC + HEMTs / } \\
\text { HEMT }\end{array}$ & ASK & $<10^{-9} /$ real-time & [9] & 10 & 2012 \\
\hline 11 & 3 & 130 & - & $\begin{array}{c}\text { 40nm CMOS } \\
(\mathrm{Tx} / \mathrm{Rx})\end{array}$ & ASK & $<10^{-9} /$ real-time & [58] & 0.033 & 2015 \\
\hline 75 & 0.02 & 200 & Frequency (3 ch) & UTC-PD / SHM & QPSK & $10^{-5} /$ off-line & [29] & - & 2014 \\
\hline 100 & 20 & 237.5 & Frequency (3 ch) & $\begin{array}{c}\text { UTC-PD / HEMT } \\
\text { Rx }\end{array}$ & $\begin{array}{l}\text { Up to QAM- } \\
16\end{array}$ & $2.10^{-3} /$ off-line & [32] & - & 2013 \\
\hline 64 & 850 & 240 & - & MHEMT - MMIC & QPSK & $5.10^{-3} /$ off-line & [61] & - & 2015 \\
\hline 64 & 1 & 300 & - & MMIC (Tx/Rx) & QPSK & - / off-line & [52] & - & 2015 \\
\hline 40 & 10 & 300 & - & UTC-PD / SHM & QPSK & $10^{-4} /$ off-line & [36] & - & 2015 \\
\hline 48 & 0.5 & 300 & Polarization (2 ch) & UTC-PD / SBD & ASK & $10^{-10} /$ real-time & [34] & 0.024 & 2013 \\
\hline 3 & 50 & 340 & - & SHM / SHM & QAM-16 & $10^{-10} /$ real-time & [65] & 0.15 & 2014 \\
\hline 32 & 0.5 & 385 & - & UTC-PD / SHM & QPSK & $10^{-5} /$ off-line & [62] & - & 2015 \\
\hline 46 & 2 & 400 & - & UTC-PD / SHM & ASK & $10^{-3} /$ off-line & [30] & - & 2014 \\
\hline 30 / 50 & $20 / 0.5$ & $300 / 330$ & - & $\begin{array}{c}\text { UTC-PD / SBD or } \\
\text { SHM }\end{array}$ & ASK & $10^{-9} /$ real-time & [28] & $0.6 / 0.025$ & 2015 \\
\hline 60 & 0.5 & 400 & Frequency (4 ch) & UTC-PD / SHM & QPSK & $10^{-3} /$ off-line & [63] & - & 2015 \\
\hline 2.5 & 3 & 625 & - & Multiplier / SBD & $\begin{array}{l}\text { Duobinary } \\
\text { (ASK) }\end{array}$ & $<10^{-9} /$ real-time & [64] & 0.0075 & 2011 \\
\hline
\end{tabular}

Table 2

\begin{tabular}{|c|c|c|}
\hline Item & Target & Technology options \\
\hline Data rate & $100 \mathrm{Gbit} / \mathrm{s} \sim 1 \mathrm{Tbit} / \mathrm{s}$ & $\begin{array}{l}\text { Multi-band (multi-carrier) system } \\
\text { Ultra-wideband optical modulators }\end{array}$ \\
\hline Link distance & $1 \mathrm{~km} \sim 5 \mathrm{~km}$ & $\begin{array}{l}\text { Integrated photodiode arrays } \\
\text { Use of amplifiers and integration }\end{array}$ \\
\hline Efficiency/cost & - & $\begin{array}{l}\text { Photonic integration } \\
\text { (III-V photonics/Si photonics) }\end{array}$ \\
\hline Key components & - & $\begin{array}{l}\text { Low-loss waveguide/interconnect } \\
\text { Wide-band/low-loss/reconfigurable antennas } \\
\text { Wide-band passive devices } \\
\text { (filter/coupler/diplexer/absorber) } \\
\text { New materials \& devices } \\
\text { (metamaterial, graphene, plasma-wave, etc.) }\end{array}$ \\
\hline Miscellaneous & - & $\begin{array}{l}\text { Propagation/interference } \\
\text { (model/real THz channels emulation/testing) } \\
\text { Standardization } \\
\text { Spectrum regulation }\end{array}$ \\
\hline
\end{tabular}

